\title{
Structure and Tribological Properties of Self - Reinforced Composite Materials Based on UHMWPE and Oxides of Titanium, Zirconium and Hafnium
}

\author{
A. M. NEMERYUK and M. M. LYLINA \\ Federal State Unitary Enterprise "State Research Institute of Chemical \\ Reagents and Pure Chemical Substances"(FSUE "IREA"),107076, \\ Moscow, Bogorodskiy val, 3, Russia. \\ ${ }^{*}$ Corresponding author E-mail: amnamn@mail.ru \\ http://dx.doi.org/10.13005/ojc/330254
}

(Received: November 20, 2015; Accepted: March 18, 2017)

\begin{abstract}
In the present study the properties and structure determination of self-reinforced materials based on ultra high molecular weight polyethylene and oxides particals of titanium, zirconium and hafnium have been carried out. tribological characteristics of these composites were also investigated.
\end{abstract}

Keywords: Self-reinforced polymer composite materials, UHMWPE, composite materials, Nanoparticles, DSC, DRS, IR, SEM, $\mathrm{TiO}_{2}, \mathrm{ZrO}_{2}, \mathrm{HfO}_{2}$

\section{INTRODUCTION}

Interest in the existing and developing polymer matrix components $(\mathrm{PC})$ is caused by the ability to control their physical-mechanical and tribological properties with respect to working conditions of friction units of various machines and mechanisms. It is successfully developing direction is considered almost one of the few ways to create functional materials, allowing to obtain products with the best performance properties. Currently, PC is widely used as structural materials and tribological replacing traditional ferrous metals and non-ferrous alloys. They play an important role in reducing the consumption of materials and machinery, to increase their reliability and durability ${ }^{1}$.

Ultrahigh molecular weight polyethylene (UHMWPE) is a product of the polymerization of ethylene, carbon-chain polymers with a molecular weight reaches millions of units. This material is characterized by high strength and tribological properties, chemical resistance. Preparation of materials based on UHMWPE containing modifying fillers in the form of ultrafine, nanoscale particles is a promising area of research, as it allows to significantly improve the performance of the composites based on UHMWPE, affecting 
the character of the supramolecular structure of the polymer, which is of interest as a basis for the development of approaches to the creation of new structural and functional materials ${ }^{2-3}$.

To PC also includes self-reinforced (selffilled), polymer composite materials, which represent a composition containing particulate fillers, or a particular class of hybrid binders based on mixtures of spatial and (or) the linear polymers. During the curing or cross-linking and formation of fragments of the polymer network in hybrid raises the thermodynamic incompatibility of the components, which leads to an unfinished microphase separation system, ie the appearance of internal interfaces. In this regard, hybrid polymer matrices are formed, which can be considered as self inforced dispersion strengthened system that is as self-polymer composite materials having basic features of the structure defined by the thermodynamic incompatibility of the components, their incomplete phase separation, and the influence of the surface of the filler. Self - inforced binders based on blends and alloys of polymers, and interpenetrating polymer networks form microfilled precipitation - reinforced structure with high physicomechanical parameters ${ }^{4}$.

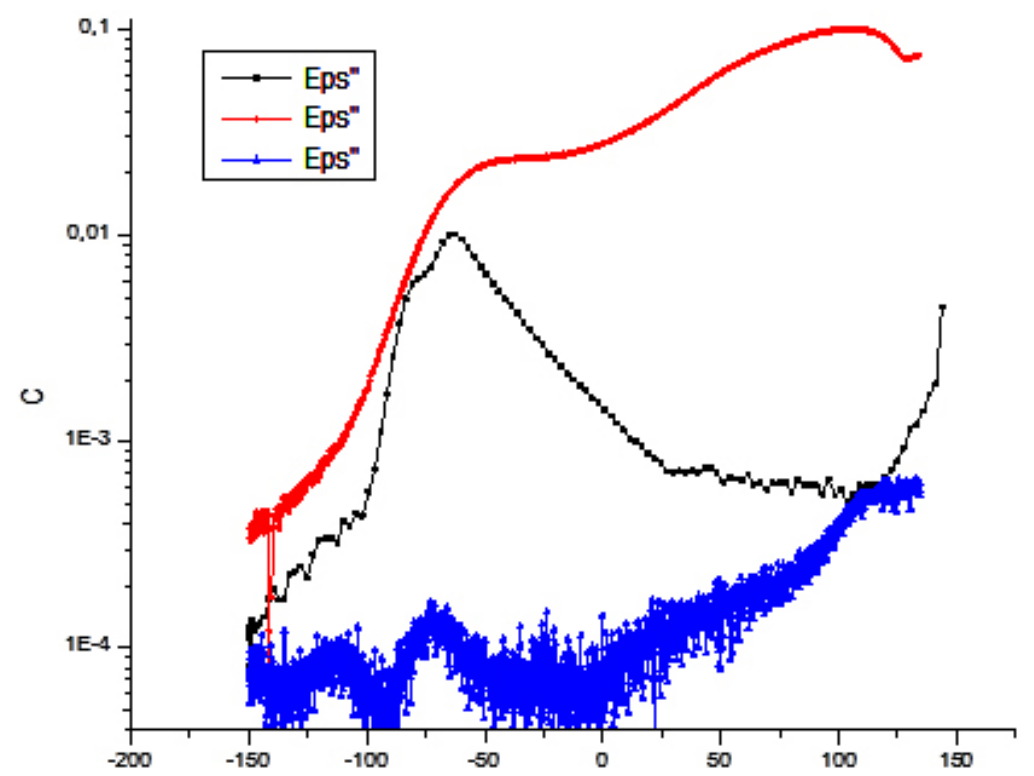

SVMPE $+\mathrm{TiO} 2,8.12 .2015,13.37$ (Fixed value(s) : Freq. [Hz]=1.100e+02 AC Volt $[\mathrm{Vm}]=1.000 \mathrm{e}+00$ )

Fig. 1: DRS - Spectrum of $\mathrm{TiO}_{2}$ self-reinforced material on the basis of UHMWPE. Blue line is starting UHMWPE.

Table 1: Data studies self-reinforced materials based on UHMWPE by DSC filler concentration $10 \%$.

\begin{tabular}{lcccc}
\hline $\begin{array}{l}\text { Name of the } \\
\text { sample }\end{array}$ & $\begin{array}{c}\text { Phase } \\
\text { transition } \\
\text { temperature } \\
\mathbf{1 , { } ^ { 0 } \mathbf { C }}\end{array}$ & $\begin{array}{c}\text { Phase } \\
\text { transition } \\
\text { temperature } \\
\mathbf{2 ,}{ }^{\circ} \mathbf{C}\end{array}$ & $\begin{array}{c}\text { Phase } \\
\text { transition } \\
\text { temperature } \\
\mathbf{3 ,}{ }^{\circ} \mathbf{C}\end{array}$ & $\begin{array}{c}\text { The } \\
\text { temperature } \\
\text { of the onset } \\
\text { of melting, }{ }^{\circ} \mathbf{C}\end{array}$ \\
\hline UHMWPE & no & no & no & 110 \\
UHMWPE $+\mathrm{TiO}_{2} 0,4 \%{ }^{*}$ & 76 & no & 100 & 116 \\
UHMWPE $+\mathrm{ZrO}_{2} 0,3 \%{ }^{*}$ & 79 & 96 & 110 & 118 \\
UHMWPE $+\mathrm{HfO}_{2} 0,5 \%{ }^{*}$ & 80 & 98 & 112 & 118 \\
\hline
\end{tabular}


Since the properties of the modified UHMWPE different from those of unmodified UHMWPE thus modified UHMWPE can be considered a self-reinforcing filler for a non-modified polymer.

\section{METHODS}

Were obtained UHMWPE powders modified with oxides of titanium, zirconium and hafnium ${ }^{2,3}$. The addition of fillers occurred by direct preparation of ultrafine or nanosized filler particles of oxide directly in the gels prepared from UHMWPE, making it possible to reach the content of metal oxides in the PC 0.1-0.5 mass\%. Powder original unmodified UHMWPE mixed in the mixer - grinder with $5-10 \%$ by weight of the modified powder. The resulting mixture was compressed on a vulcanizing thermopression $250 \times 250 \mathrm{~mm}$ at a temperature of

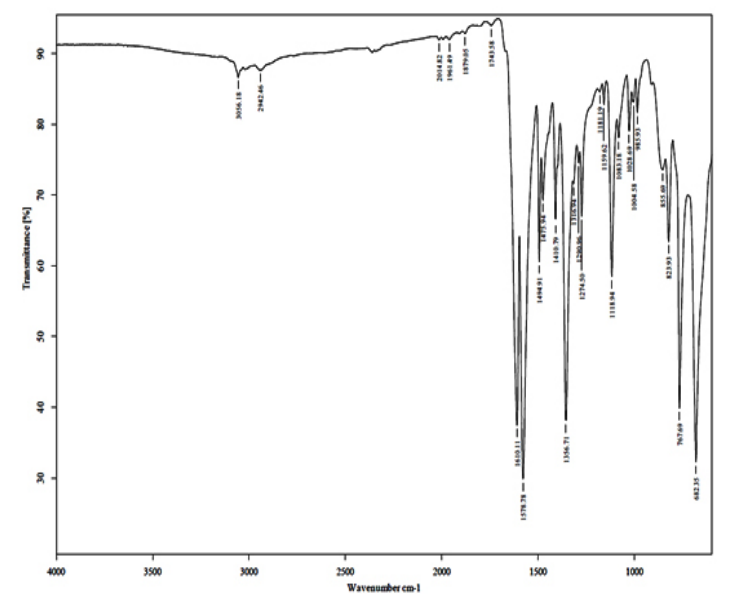

Fig. 2: IR - spectrum of $\mathrm{TiO}_{2}$ self-reinforced material on the basis of UHMWPE $200^{\circ} \mathrm{C}$ for 15 minutes at $10 \mathrm{MPa}$, followed by cooling at $3-4{ }^{\circ} \mathrm{C} /$ minutes.

The samples obtained composite self-reinforced materials based on UHMWPE containing nanoparticles of titanium, zirconium and hafnium oxides have been investigated by various methods, including differential scanning calorimetry (DSC), dielectric relaxation spectroscopy (DRS), infrared spectroscopy (IR),) and scanning electron microscopy (SEM). These methods allow to obtain data about the material, showing its structure and properties, including the presence or absence of phase transitions in the temperature range covered by the working range of the devices used for the study ${ }^{5}$. Direct and indirect data describing the composition and structure of materials allow to identify patterns associated with the peculiarities of the influence of the conditions for obtaining

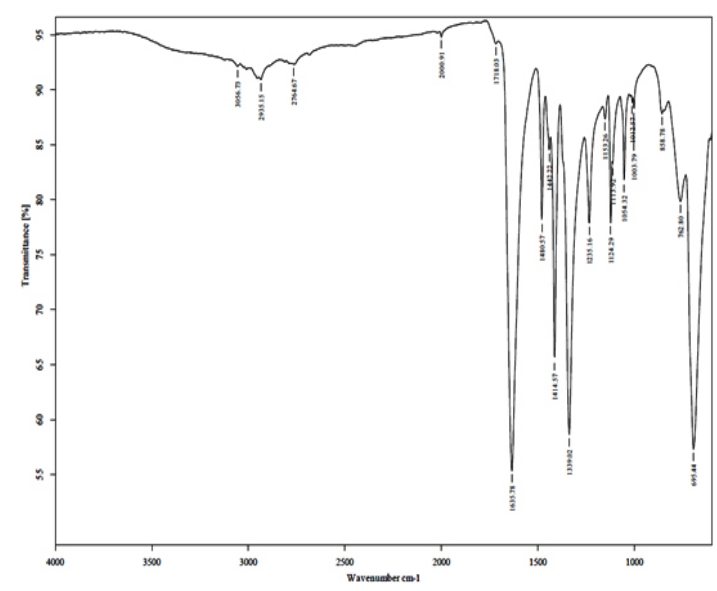

Fig. 3: IR - spectrum of $\mathrm{ZrO}_{2}$ self-reinforced material on the basis of UHMWPE

Table 2 : Data studies filler materials based on UHMWPE by DSC

\begin{tabular}{lcccc}
\hline $\begin{array}{l}\text { Name of the } \\
\text { sample }\end{array}$ & $\begin{array}{c}\text { Phase } \\
\text { transition } \\
\text { temperature } \\
\mathbf{1 ,}{ }^{\circ} \mathbf{C}\end{array}$ & $\begin{array}{c}\text { Phase } \\
\text { transition } \\
\text { temperature } \\
\mathbf{2},{ }^{\circ} \mathbf{C}\end{array}$ & $\begin{array}{c}\text { Phase } \\
\text { transition } \\
\text { temperature } \\
\mathbf{3},{ }^{\circ} \mathbf{C}\end{array}$ & $\begin{array}{c}\text { The } \\
\text { temperature } \\
\text { of the onset } \\
\text { of melting, }{ }^{\circ} \mathbf{C}\end{array}$ \\
\hline UHMWPE & no & no & no & 110 \\
UHMWPE $+\mathrm{TiO}_{2} 0,1 \%$ & 76 & 89 & 98 & 112 \\
UHMWPE $+\mathrm{ZrO}_{2} 0,1 \%$ & no & 91 & 100 & 114 \\
UHMWPE $+\mathrm{HfO}_{2} 0,1 \%$ & no & 93 & 101 & 116 \\
\hline
\end{tabular}


composite nanomaterials on their morphological characteristics and chemical.

\section{RESULTS and DISCUSSIONS}

Research conducted on a differential scanning calorimeter DSC 8500, 2010, Perkin Elmer. In the course of investigations by DSC revealed phase transitions in self-reinforcing materials based on UHMWPE containing nanoparticles of transition metal oxides, not peculiar to the starting UHMWPE. Thus is confirmed the previously expressed position on the impact of nano-sized additives on the supramolecular structure of UHMWPE, reflected in the increase in the degree of crystallinity, which has a beneficial effect on the properties of materials, as most of the ordering of packaging macromolecules polymer promotes the values of the strength,

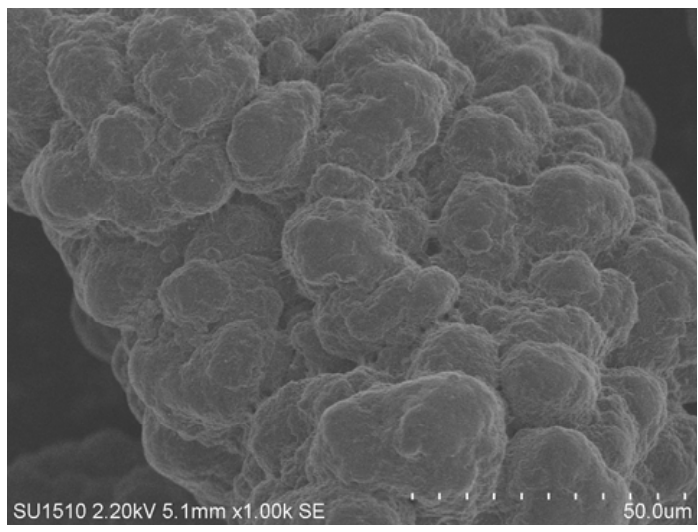

Fig. 4: The resulting SEM image of a sample of the original UHMWPE

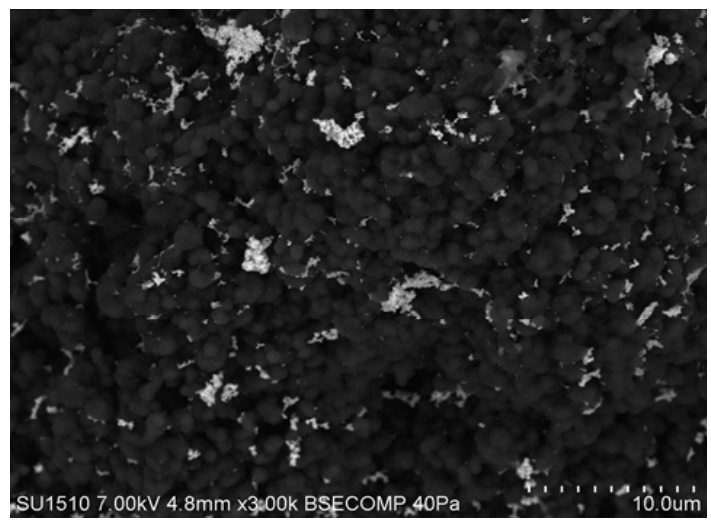

Fig. 6: The resulting SEM image of a sample of UHMWPE filer containing nanoparticles of zirconium oxide abrasion resistance ${ }^{6}$. Of particular note is the growth temperature of the onset of melting materials based on UHMWPE containing nanoparticles of oxides of zirconium and hafnium, and a more pronounced influence of hafnium oxide nanoparticles that can be attributed to a greater polarization of the surface of these particles. Table 1 summarizes the data obtained during the study of samples of UHMWPEbased materials containing oxide Nanoparticles by DSC. Table 2 summarizes the data obtained during the study of fillers samples by DSC.

According to Table 1, in self-reinforced materials containing the oxides of zirconium and hafnium, a phase transition at $96-98^{\circ} \mathrm{C}$ is absent in the starting UHMWPE samples and self-reinforced materials containing titanium dioxide particles. Also an increase in the onset temperature of melting at

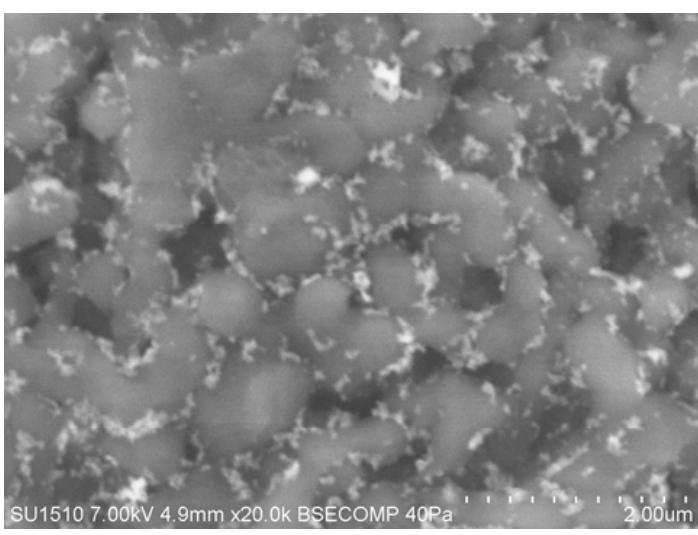

Fig. 5: The resulting SEM image of a sample of UHMWPE filer containing nanoparticles of titanium oxide

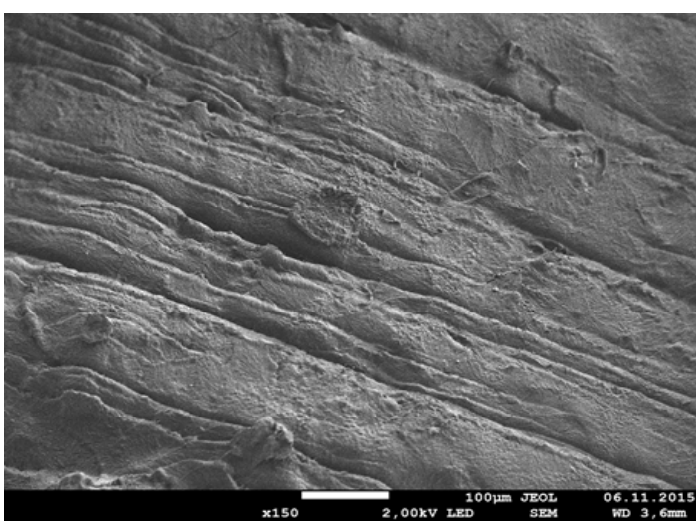

Fig. 7: The resulting SEM image of a sample of UHMWPE, self-reinforcing composite containing nanoparticles of zirconium oxide 
the transition from the oxides of titanium to oxides of hafnium, with an equal percentage, indicating the greater effect of hafnium oxide on the crystallinity of UHMWPE.

In the study of the substance has been identified by the DRS dielectric loss tangent at certain temperatures. UHMWPE samples containing the metal oxides as well as starting UHMWPE were investigated by DRS in the temperature range from $-170^{\circ} \mathrm{C}$ to $150^{\circ} \mathrm{C}$.

In general, the spectra of the DRS for example figure. 1 spectrum of $\mathrm{TiO}_{2}$ self-reinforced material on the basis of UHMWPE and the other oxides modified UHMWPE starting material have similar features, but the dielectric loss tangent at temperatures from $-80^{\circ} \mathrm{C}$ to $-50^{\circ} \mathrm{C}$ is more important, reaching $1^{\star} 10^{-5}$ for samples containing hafnium oxide, slightly lower Dielectric loss values are determined for samples with nanoparticles of titanium oxide and zirconium oxide, whereas the starting UHMWPE dielectric loss tangent within this temperature range an order of magnitude less. Similar values of dielectric loss tangent obtained at temperatures of $60^{\circ} \mathrm{C}$ to $90^{\circ} \mathrm{C}$. At the moment the theoretical basis of the data insufficiently formulated and is being studied. However, increasing the scattering of electromagnetic energy in the medium UHMWPE samples containing oxides of titanium, zirconium and hafnium indicates a greater polarizability of the medium, due to the presence of oxide particles. Also, the dependence of the dielectric loss tangent of the chemical nature of the filler, in the presence of hafnium oxide value of the dielectric loss tangent is higher than in the presence of oxides of zirconium and titanium, which is correlated with the data of DSC, demonstrating a greater impact on the hafnium oxide supramolecular polymer packaging. The latter relates to the degree of polarization of the surface of the filler particles. Thus, the data obtained by DRS confirm a theoretical model relating the degree of polarization of the surface of the filler particles with the influence on the properties of the polymer matrix ${ }^{7}$. This is also true for self-reinforced samples, where the filler is applied on the basis of highly composite UHMWPE and hafnium oxide nanoparticles.

The studies by IR spectroscopy was used to confirm the absence of organic side products in the polymer matrix UHMWPE self-reinforcing oxi de nanoparticles of titanium, zirconium and hafnium composites. Figure 1 shows the IR - spectrum self-reinforcing materials based on UHMWPE. The spectrum was obtained on a IR - Fourier spectrometer Bruker VERTEX 70.

The resulting spectrum was identical to the IR - spectrum of the starting UHMWPE, which indicates the absence of organic impurities that can absorb electromagnetic energy in the infrared spectrum.

During the investigation of the structure self-reinforced materials based on UHMWPE containing oxides of titanium, zirconium and hafnium was examined by SEM surface of the test specimens, as well as the original unmodified SVMP. Figures 4 is a SEM image of a sample of the original UHMWPE, 5-6 UHMWPE with metal oxides samples, 7 are images self-reinforced UHMWPE sample containing filler with nanoparticle oxides, scanning electron microscope Hitachi SU1510 and JEOL 7200F.

From the image you can see that there is a significant change in the nature of the polymer surface of the grain, which is due to mechanochemical phenomena that take place during the formation of self-reinforcing materials using crushing and mixing equipment.

Experimental samples and original nonmodified UHMWPE were used for tribological testing, during which a determined coefficient of dry friction. Scheme sample loading was the use of the $1 \mathrm{~cm}$ diameter cylinder experimental sample fixedly mounted in contact with the rotating plate made of steel $\mathrm{SH}-15$ under the application of external force. The velocity of the opposing member was $2.5 \mathrm{~m} / \mathrm{s}$, the load at the point of contact - $2 \mathrm{~kg}$, the medium - air, room temperature $\left(22-24^{\circ} \mathrm{C}\right)$. The test was continued for $20 \mathrm{~h}$, not including time prior reaction for $30 \mathrm{~min}$. required for grinding test sample to counterbody. It was shown that the samples containing as a filler particles of titanium oxide have dry friction coefficient of 0.8 , and samples containing particles of oxides of zirconium and hafnium are characterized by an indicator of dry friction of 0.7 , whereas the figure for the non-modified sample of UHMWPE was 0.11 . 


\section{CONCLUSION}

In the structural studies of the self-reinforced composite material containing nanoparticles of titanium oxides, zirconium and hafnium $\left(\mathrm{TiO}_{2}, \mathrm{ZrO}_{2}\right.$, $\mathrm{HfO}_{2}$ ) by DSC, DRS, IR, and SEM were examined properties of the obtained experimental samples and a number of intermediates. DSC were found new phase transitions in materials containing oxide nanoparticles show an increase in the onset temperature of melting. The method of the DRS determined the dielectric loss tangent in the material, found a marked increase in the dielectric loss of the samples containing metal oxides, the observed correlation between the properties of the materials so increasing the dielectric loss as much as possible in samples containing hafnium oxide, they are also inherent in the maximum temperature rise of the beginning melting. By IR - spectroscopy confirmed the absence of impurities of organic compounds in samples the self-reinforced composite materials based on UHMWPE.
SEM was used to study the surface of the experimental self-reinforced UHMWPE samples containing oxides of titanium, zirconium and hafnium. It is shown that there is a significant change in the nature of the polymer surface of the grains.

It is shown that the self-reinforced samples containing oxide particles have a lower coefficient of friction than unmodified samples of UHMWPE.

\section{ACKNOWLEDGEMENTS}

Applied researches are carried out with financial support of the state by the Russia Ministry of Education and Science under Grant Agreement 14.576.21.0004 of June 17, 2014. (Unique identifier for Applied Scientific Researches (project) RFMEFI57614X0004).

\section{REFERENCES}

1. Danilov V.D. Journal of scientific and technological development, 2008, 12(16), 14-19

2. Nemeryuk A, Sudarikova E, Lylina M, Romanova E, Ramsh A, Omelchenko A. Chemical industry today, 2013, 12, 27-37

3. Nemeryuk A, Sudarikova E, Lylina M, Romanova E, Zhdanovich O. Chemical industry today, 2013, 11, 25-28

4. Seljutin G.E., Gavrilov Y.Y., Voskresenskaya E.N., Zakharov V.A., Poluboyarov V.A. Chemistry for Sustainable Development,
2010, 18, 375-388

5. Sutyagin V.M., Lyapkov A.A., Physical and chemical research methods of polymers. Tomsk Polytechnic University Publishing House, Tomsk (2008)

6. Panin S.V., Panin V.E., Kornienko L.A., Puvadin T., Piriyaon S., Shilko S.V., News of Higher Schools. Series: Chemistry and Chemical Technology, 2011, 54 (7), 102106

7. Pomogailo A.D. Metal nanoparticles in polymers. Chemistry, Moscow (2000) 